\title{
Avoiding Porosity in Gold Plating
}

\section{A NEW METHOD OF STUDYING DEFECTS AND THEIR CAUSES}

\author{
H. S. Campbell \\ The British Non-Ferrous Metals Research Association, Lonidon
}

\begin{abstract}
The essential part played by electrodeposited gold in telecommunications and computers, and in electrical and electronic equipment generally, depends upon the production of deposits suffciently free from pores or other defects to maintain a clean, corrosion resistant surface. The use of a scanning electron microscope to investigate porosity in gold plating has emphasised the importance of ensuring the absolute cleanliness of the surfaces to be plated.
\end{abstract}

The resistance of gold to tarnishing and corrosion makes it outstanding as a material for electrical contacts in electronic components. For this purpose it is employed as plating-usually on copper or a copper-rich alloy. It is, of course, essential that the plating be sufficiently free from pores or other defects to exclude corrosion of the underlying metal and consequent formation of corrosion products that can spread over the gold surface and increase the electrical resistance of the contact surface. To achieve the high degree of integrity in the plating that this implies, a minimum plating thickness of 5 micrometres is normally specified but, even at that thickness, great care is required, both in plating and in preparation for plating, to ensure freedom from porosity.

The British Non-Ferrous Metals Research Association has been concerned for several years in studies of the causes of porosity in gold plating and of means to prevent it. Work carried out in the Association's laboratories by Ashurst \& Neale, published in 1967, showed the improvement that could be obtained by avoiding certain types of polishing compound in the preparation stages, by chemical polishing or electropolishing before plating and by using a nickel undercoat beneath the gold. It was not clear, however, at that stage, whether the porosity that still occurred rather unpredictably in deposits laid down using the best commercial practice was due to surface con- tamination that was present in spite of the care taken, or to the effect of hydrogen bubbles forming on the surface during plating or to some other cause. The path towards further improvement was, therefore, uncertain.

It was shown later, by electrochemical studies in which the plating current density, the nature of the substrate and other factors were varied, that the production of porosity was not associated with the formation of hydrogen bubbles, but it was more difficult to investigate the possible effects of contamination and surface defects in the substrate on which the gold is plated. It was obvious that the best way would be by direct observation of the pores at high magnification but the practical difficulty of doing this lies in the fact that the pores are extremely small, requiring a magnification of around 5,000 times to study them in any detail, and are very few and far between on the plated surface. Ordinary microscopes will not provide the necessary magnification; electron microscopes will, but the normal type cannot be used for direct examination of the plated surface. A thin plastic film replica of the surface has to be examined instead and the chance of a pore being included in the very small area covered by the replica is low.

These difficulties have been overcome by the use of a Scanning Electron Microscope, one of the newest instruments for the detailed study of surfaces. With the Scanning Electron Microscope (SEM) it is possible to scan rapidly the whole surface of a specimen up to $7 \mathrm{~mm}$ square, to locate any pores or other features of interest and then to switch to higher magnification and examine them in detail. Another very important advantage of the SEM over the conventional electron microscope is its unusual depth of focus which provides a three-dimensional view of pores, much more informative than can be obtained by other means.

Using an SEM, we have observed two types of pore in gold electrodeposits. Those of the first type were of irregular shape, very numerous in deposits up to about 0.2 micrometres thick, but not present in thicker deposits. Those of the second type were roughly triangular in shape, fewer but larger than those of the first type and persisting even in the thicker deposits. Fig. 2 is an SEM photograph of a gold deposit 0.1 micrometres thick, showing one large 


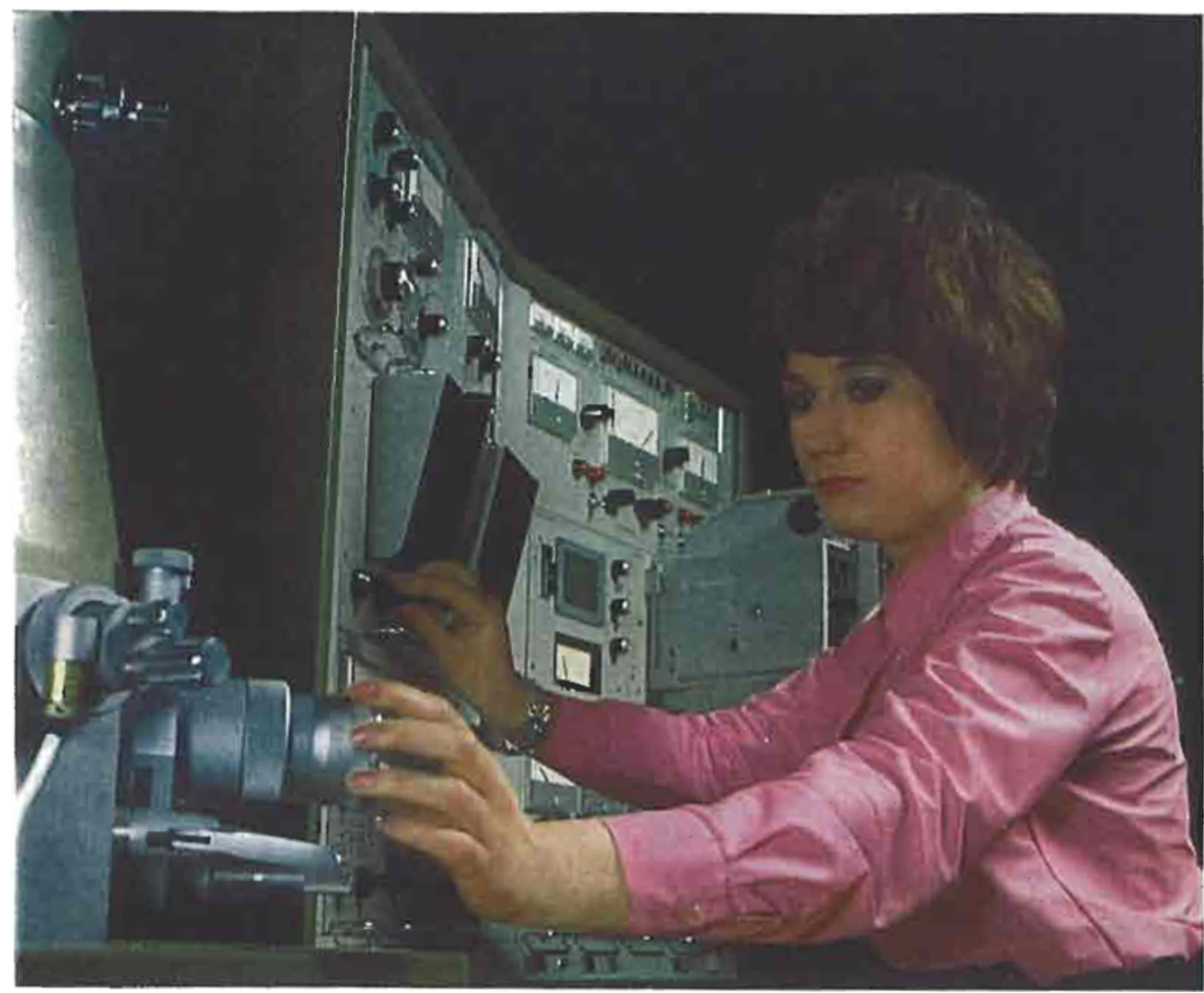

Fig. I The Cambridge Scaming Electron Microscope for the detailed study of surfaces used to investigate the nature of the pores occurring in gold electrodeposits. The advantage of this instrument over the conventional electron microscope resides in its unusual depth of focus, which provides much more information in a three-dimensional view of any defects

triangular pore and numerous small irregularly shaped pores. A prominent feature frequently observed in the triangular pores is the apparently crystalline particle which shows up darker than the gold in the lower right-hand corner of the pore.

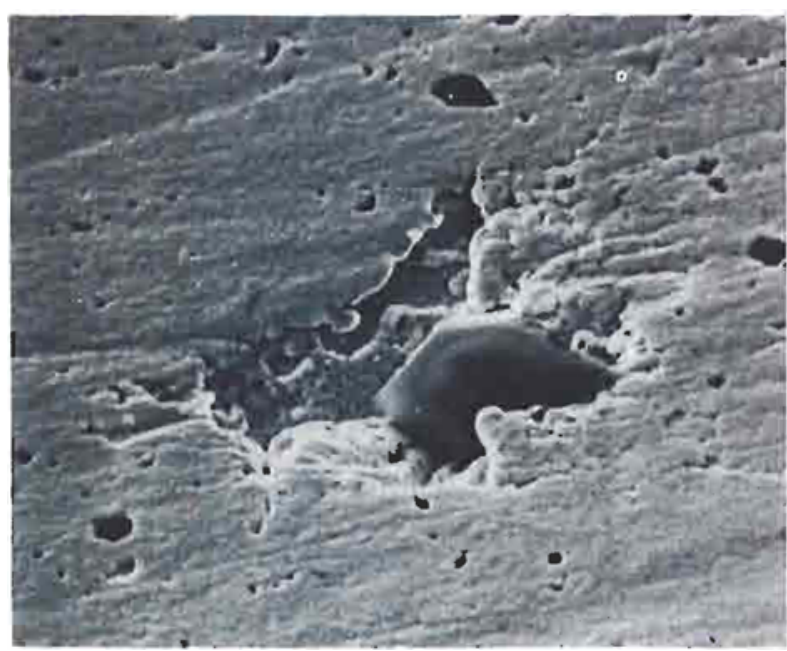

Fig. 2 Small irregular pores and a larger triangular pore in very thin $(0.1$ micrometre) gold plate. Note the particle of residual polishing compound in lower right-hand corner of the pore.
Fig. 3 shows another triangular pore in a thin gold deposit but this time with two or three inclusions present in it. Along the top edge of the pore a flap of gold is seen growing parallel to the surface. Presumably, if the deposition of gold had been continued,

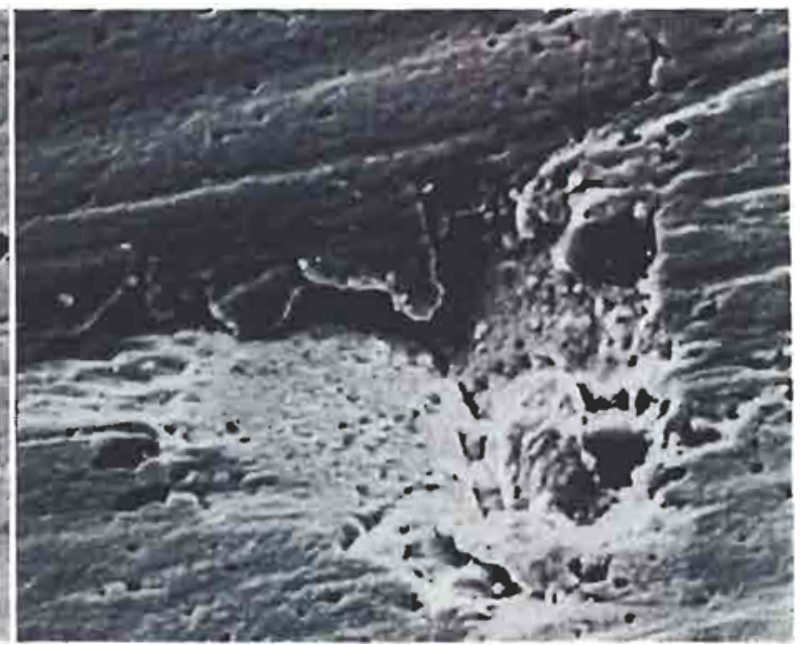

Fig. 3 A flap of gold growing out over a triangular pore in 0.1 micrometre gold plate. The pore would eventually be covered over but not filled up by the gold. $\quad(\times 5500)$ 


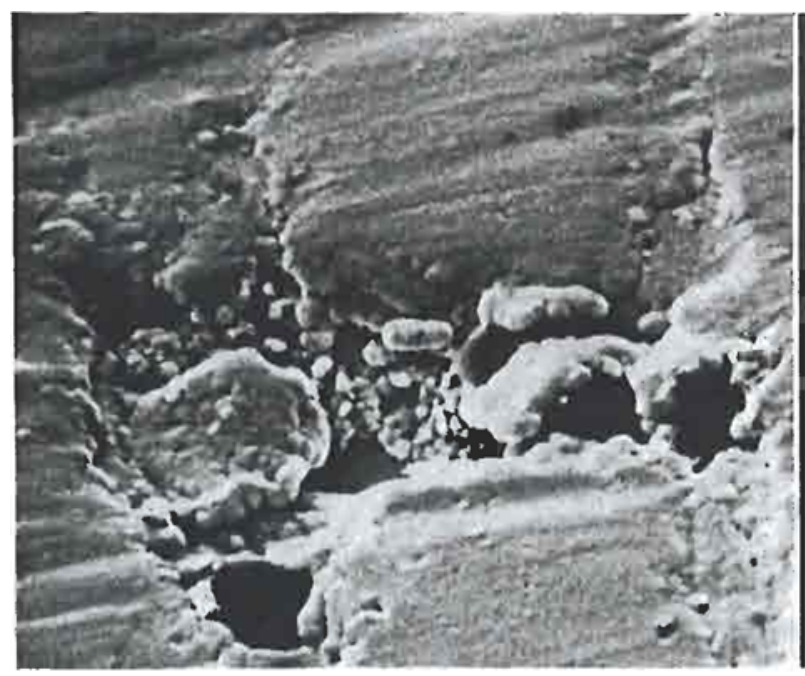

Fig. 4 Gold beginning to spread over a partiele of residual polishing compoumd in a triangular pore in 0.2 micrometre gold plate. The gold would eventually fill the pore but prohably only as a rathex spongy deposit giving unreliahle protection.

$(\times \mathbf{5 7 5 0})$

this would have grown right across the pore but there would still have been a gap beneath it. Fig. 4 illustrates another way in which gold gradually covers over a pore as the thickness of the plating builds up. The pore in Fig. 4 contains one small inclusion at the lowest point of the triangle and another in its top left-hand corner but in the right-hand corner is a rather large inclusion with gold beginning to deposit over it. The feature projecting from the left-hand side of the triangle may be a fourth inclusion almost completely covered by gold, or it may be a flap of gold growing out across the pore, similar to that shown in Fig. 3. Whichever mechanism results in eventual covering of the pore by gold, there will still be a local defect and possibly even a group of minute discontinuities in the plating where the pore was originally located.

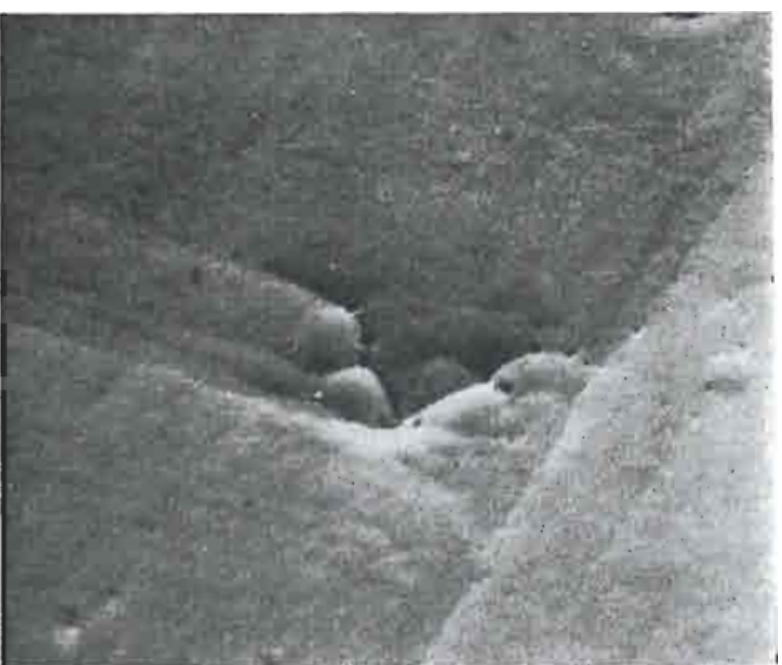

Fig. 5 A gold deposit 2.0 micrometres thick which has eovered over a pore that was present when the plating was thinner but still shows obvious weakness where the pore was situated.

$(\times \mathbf{4 5 0 0})$

Fig. 5 shows a much thicker (2 micrometre) deposit; the feature at the centre of the photograph is probably one of the triangular pores of the type shown in Figs 2, 3 and 4 now largely covered by gold but still clearly a weak point in the plating.

The SEM that was used for studying pores in gold plating incorporated a facility for making X-ray analyses on selected minute areas of specimens while in the microscope. Using this, it was shown that the inclusions associated with triangular pores were particles of polishing compound still remaining in spite of the elaborate cleaning procedure that had been adopted before plating. The difficulty of preparing surfaces free from such contamination by the normal commercial pre-plating cleaning procedures is obvious and further work is, therefore, being carried out on improved cleaning methods.

\section{Structure of the Gold-Silicon Eutectic}

The low melting point eutectic alloys of gold-silicon, gold-germanium and gold-tin are used as solders in electronic engineering to make electrical or thermal connection between a silicon chip and a suitable substrate, the chip being a pre-alloyed or diffused semi-conductor diode, transistor or integrated circuit. In the case of gold-silicon-the eutectic composition is at 3.25 per cent gold and the melting point $370^{\circ} \mathrm{C}-$ the eutectic is either used as a pre-form produced from strip or it is developed in situ by depositing gold on to the silicon and alloying at a temperature above the eutectic.

Studies of the microstructure of this alloy and of its modification by varying the rate of cooling from the liquid state were reported by E. Philofsky and his collaborators of Motorola Semiconductor Products to the meeting of the Electrochemical Society in October last. Cooling rates ranging from less than $\mathrm{Io} \mathrm{deg} . \mathrm{C} / \mathrm{sec}$ to more than $1000 \mathrm{deg} . \mathrm{C} / \mathrm{sec}$ were used. At the slowest rates a coarse microstructure was found comprising discrete silicon particles of various shapes in a gold matrix. At intermediate rates a cylindrical dendrite structure was observed, the dendrite spacing decreasing as cooling rate increased, while at the very high rates of cooling-obviously impracticable in actual semiconductor techniques-X-ray diffraction revealed the presence of new complex cubic structures in addition to gold and silicon. Subsequent heat treatment transformed this structure to one consisting apparently of pure gold with the silicon too fine to resolve.

These findings could possibly be helpful in the fabrication of gold-silicon eutectic alloy strip from its brittle as-cast structure by appropriate heat treatment followed by cold rolling. 